Pensamiento Crítico Vol. 21 No 1, pp. 7 - 24

\title{
El interés de China en Latinoamérica
}

\author{
China interest in Latin America
}

[Recepción: Marzo 2016 / Conformidad: Mayo 2016]

Carlos Aquino Rodríguez ${ }^{1}$

\section{RESUMEN}

El interés de China por Latinoamérica ha aumentado. Tiene una estrategia para incrementar su presencia, y planea duplicar el comercio y la inversión con la región para el 2025.

En el presente artículo, se analizan los últimos desarrollos concernientes a esos temas y en la primera parte se verá el caso del Perú, país de una antigua relación con China, donde se ve un creciente interés chino en los sectores extractivos. También se ve cómo, dada la creciente presencia china en el Perú, se podría convertir en un hub de este país en la región. Asimismo, se ve que China está mostrando un nuevo interés para invertir en otras áreas en la región, como en la infraestructura física, energía nuclear y agricultura.

Palabras clave: Interés de China en Latinoamérica, Relaciones económicas entre China y Latinoamérica, Inversión china en Latinoamérica.

1 Con estudios de maestría y doctorado en la Universidad de Kobe, Japón. Profesor de la Facultad de Economía de la UNMSM. Especialista en Economía y Política Asiática. Director del Instituto de Investigaciones Económicas. E-mail: carloskobe2005@yahoo.com 


\title{
Pensamiento Crítico Vol. ᄅl No I
}

\begin{abstract}
The interest of China in Latin America has increased and it is planning to double its trade and investment with the region by the year 2025 .

In this article we analyze the latest developments regarding China interest in the región and in the case of Peru we see an increasing interest of China in investment in the primary sector, Also the possibiity of Peru becoming a hub of China in the region is analyzed. Besides, the growing interest of China in investment in other áreas, as in infrastructure, nuclear energy and agriculture is showed.
\end{abstract}

Keywords: China interest in Latin America, Trade relations between China and Latin America, China investment in Latin America.

\section{Introducción}

China en los últimos años ha incrementado sus relaciones económicas y su interés en Latinoamérica. Su comercio con la región, sus inversiones y el financiamiento que da a algunos países se ha incrementado notablemente.

En el presente artículo se analizarán los últimos desarrollos concernientes a esos temas y en la primera parte se verá el caso del Perú, país de una antigua relación con China, tocando en detalle el caso de las inversiones chinas en el país, especialmente el de la minería. En la segunda parte, se verá si el Perú puede convertirse en un hub para China en la región. En la tercera parte, se ve algunas áreas donde China ha mostrado un nuevo interés para invertir, como en la infraestructura física, energía nuclear y agricultura. En la cuarta parte se entregan las conclusiones.

El interés de China por Latinoamérica ha aumentado y también de la región por China. Este país tiene una estrategia para incrementar su presencia y planea duplicar el comercio a 500 mil millones de dólares y la inversión hasta 250 mil millones de dólares en la región en unos 10 años (al 2025).

El comercio de China con la región se incrementó grandemente, unas 22 veces, entre el 2000 y el 2014 como se ve en el Gráfico 1, y actualmente el gigante oriental es el tercer destino de las exportaciones de la región, después de EE.UU. y Europa, y la 


\section{Carlos Aquino Rodríguez}

segunda fuente de importaciones después de EE.UU. Ver Gráfico 2. Pero si uno omite a México, que comercia bastante con EE.UU., China en realidad es ya el segundo socio comercial de Latinoamérica y el primero de varios de ellos.

El presidente chino Xi Jinping, durante el Primer Foro de la Comunidad de Estados Latinoamericanos y Caribeños (CELAC)-China realizado en Beijing en enero del 2015², y el primer ministro Li Keqiang, durante su visita a la región en mayo del $2015^{3}$, reiteraron el interés de China y su propósito de incrementar no solo las relaciones económicas, sino de cooperación para el desarrollo de la región, así como también de intercambio de experiencias de desarrollo e intercambio de personas.

\section{GRÁFICO 1}

Comercio en bienes de China con Latinoamérica, 2000-2014 (millones de dólares)

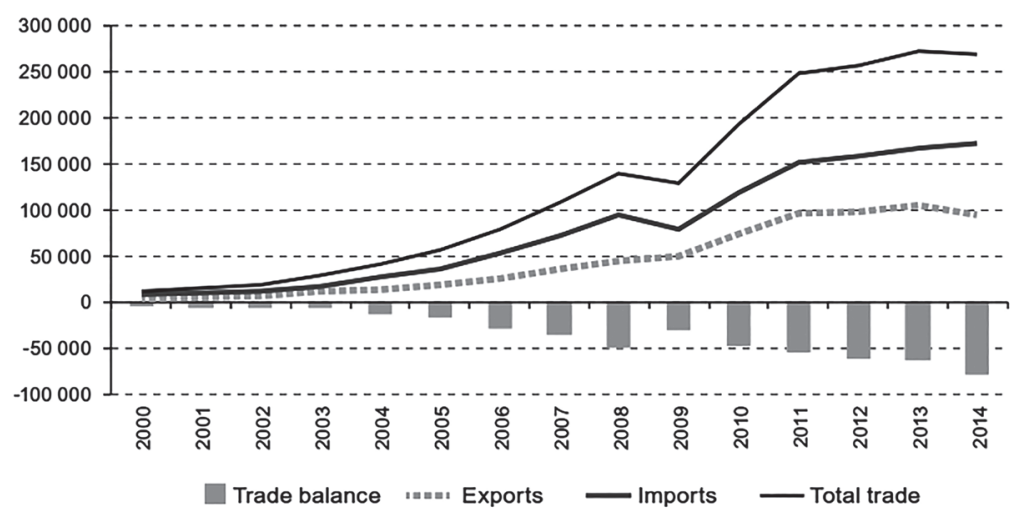

Fuente: CEPAL, mayo del 2015: "Latin America and the Caribbean and China: towards a new era in economic cooperation"http:/www.cepal.org/en/publications/38197-latin-america-and-caribbean-andchina-towards-new-era-economic-cooperation, pagina 36

2 Ver diario El Universal versión online: "En reunión China-Celac se acordó un plan quinquenal de cooperación" http://www.eluniversal.com/nacional-y-politica/150109/en-reunion-china-celac-se-acordo-unplan-quinquenal-de-cooperacion(accedido el 15 de febrero del 2016)

3 Ver la noticia de su visita y su discurso en la sede central de la CEPAL en Chile: "China BetsOnStrategicTieswithLatinAmerica and theCaribbean" http://www.cepal.org/en/pressreleases/china-apuesta-porvinculo-estrategico-con-america-latina-y-el-caribe(accedido el 15 de febrero del 2016) 


\section{Pensamiento Crítico Vol. ᄅl $\mathrm{N}^{\circ}$ I}

En momentos en que la baja en los precios de los recursos naturales, que constituyen casi un $70 \%$ del total que exporta la región a China, ha afectado fuertemente a Latinoamérica, China ofrece fortalecer el aparato productivo e industrializarlo. La razón también de esto es que China es sensible a las críticas de que el patrón de comercio entre ese país y Latinoamérica es asimétrico, donde la región mayormente le vende materias

primas y obtiene a cambio productos manufacturados. Asimismo, se ha señalado que las inversiones chinas, centradas mayormente en la extracción de recursos naturales, refuerzan ese patrón de comercio asimétrico.

\section{GRÁFICO 2}

Porcentaje de comercio en bienes de Latinoamérica con algunos socios comerciales Exportaciones e importaciones, 2000-2014
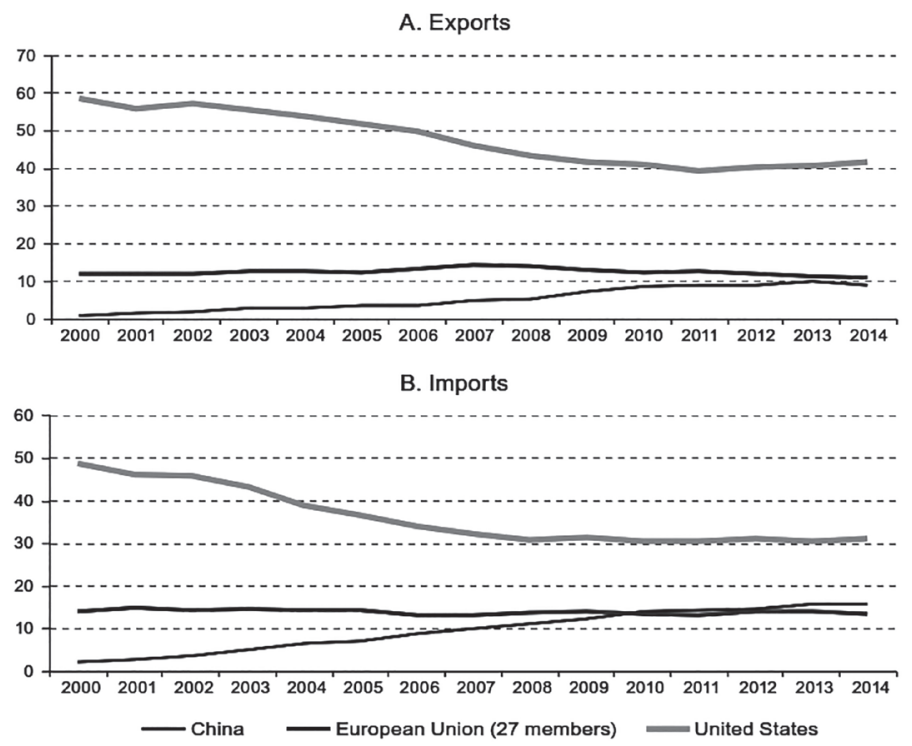

Fuente: CEPAL, mayo del 2015: "Latin America and the Caribbean and China: towards a new era in economic cooperation"http:/www.cepal.org/en/publications/38197-latin-america-and-caribbean-andchina-towards-new-era-economic-cooperation, Página 37

El interés chino en Latinoamérica es porque esta es una fuente de materias primas, desde minerales, hidrocarburos, hasta alimentos. Pero también la región ofrece un mercado para los productos chinos y un destino para sus empresas, que con la menor 


\section{Carlos Aquino Rodríguez}

tasa de crecimiento económico en su país ahora tienen una capacidad instalada ociosa. Además, es un objetivo de China la internacionalización de sus empresas para ser más competitivas.

Dentro de la región, hay algunos países con los que tiene una fuerte relación, como con Perú y Chile, con los que tiene firmados tratados de libre comercio. En el caso del Perú, con el cual tiene una relación muy antigua y donde hay una comunidad grande de descendientes chinos, y siendo un país que posee grandes recursos naturales, la presencia china va en aumento. El régimen económico peruano, de bastante apertura a la inversión y comercio con el extranjero, ha hecho posible que China sea su primer socio comercial, y que la inversión china en minería e hidrocarburos en particular sea cada vez más importante. Por ejemplo, una empresa china produce el $100 \%$ del hierro del Perú, con sus nuevas inversiones en las minas de cobre sus empresas producirían un $30 \%$ o más del total de este producto, y una empresa petrolera producirá un $30 \%$ a 40\% del petróleo y gas del país.

A pesar de la desaceleración de su economía, China seguirá necesitando recursos naturales y sus empresas saldrán con más fuerza al exterior. Nuevas áreas de interés de China son la infraestructura física (provisión de trenes, por ejemplo), la energía nuclear, la agricultura. China puede ofrecer condiciones favorables de financiamiento para proyectos, lo cual la pone en ventaja frente a otros competidores.

China, como se sabe, ha establecido mecanismos para poder financiar estos proyectos. Tiene el recién creado Banco Asiático de Inversión en Infraestructura, BAII, y el Nuevo Banco de Desarrollo de los BRICS, que quieren financiar la construcción de la infraestructura física de los países en desarrollo, empezando por los de Asia, pero también tiene el objetivo de la construcción de la ruta económica y marítima de la seda, que uniría China con el Asia, Europa y África ${ }^{4}$. Aparte de esto, China, a través de sus propios bancos e instituciones financieras, como el China Development Bank y el Export-Import Bank, ha prestado bastante dinero a Latinoamérica.

Por ejemplo, el año 2015 China financió a la región por 29 mil millones de dólares, más que los 19 mil millones de dólares que dio el 2014. Como dice un medio, "De nuevo,

4 Ver por ejemplo páginas 30 y 31 del estudio de la CEPAL de mayo del 2015: "Latin America and the Caribbean and China: towards a new era in economic cooperation" http://www.cepal.org/en/ publications/38197-latin-america-and-caribbean-and-china-towards-new-era-economic-cooperation (accedido el 15 de febrero del 2016) 


\section{Pensamiento Crítico Vol. ᄅl $\mathrm{N}^{\circ}$ I}

y como ha venido ocurriendo en los últimos años, la inversión de China en Latinoamérica superó los préstamos conjuntos aportados por el Banco Mundial (BM), el Banco Interamericano de Desarrollo (BID) y el Banco de Desarrollo de América Latina (CAF)". ${ }^{5}$

China presta bastante, especialmente, a países en la región que no pueden acceder al crédito externo, pues están excluidos del sistema financiero internacional, como Argentina, Venezuela y Ecuador. Así, "El Banco de Desarrollo de China (BDC) prestó el año pasado la friolera de 10,000 millones de USD a Venezuela -un tercio del total de los préstamos a Latinoamérica y el Caribe- a pesar del creciente riesgo de una cesación de pagos por el país petrolero" 6 . Se dice que China ha prestado a la región más de 125 mil millones de dólares desde el 2005, y un tercio ha ido a Venezuela. Ver Gráfico 3.

\section{GRÁFICO 3}

Préstamos de China a Latinoamérica en el 2015 y préstamos a Venezuela y

Latinoamérica en el periodo 2005-2015

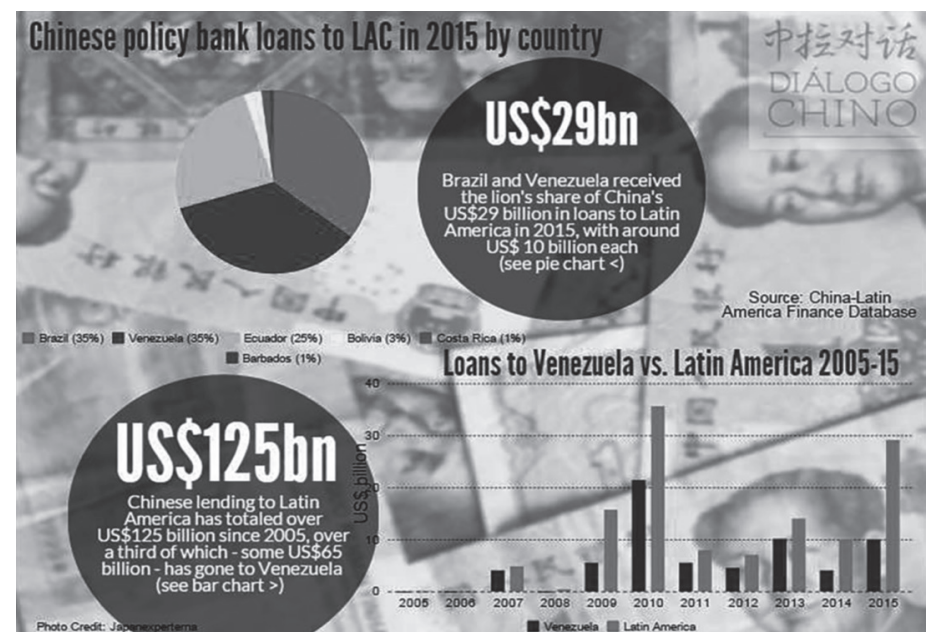

Fuente: Ver Diálogo Chino: "China aumenta préstamos a Latinoamérica" http://dialogochino.net/china-aumenta-prestamos-a-america-latina/?lang=es

5 Ver eleconomista.es: "China redoblo la inversión en Latinoamérica y se centra en infraestructuras" http:// www.eleconomista.es/economia/noticias/7345706/02/16/China-redobla-la-inversion-en-Latinoamerica-yse-centra-en-infraestructuras.html(accedido el 15 de febrero del 2016)

6 Ver Diálogo Chino: "China aumenta préstamos a Latinoamérica" http://dialogochino.net/china-aumentaprestamos-a-america-latina/?lang=es(accedido el 15 de febrero del 2016) 


\section{Carlos Aquino Rodríguez}

Por otro lado el turismo chino, que cada vez crece más, también mirará a la región, que, aunque lejana, ofrece lugares interesantes que visitar, como México, Perú y otros. Los chinos son los turistas con la mayor capacidad de poder adquisitivo en la actualidad.

\section{Creciente inversión China en el Perú}

El Perú tiene abundantes recursos mineros, energéticos y tierra que puede ser convertida a la agricultura. Su régimen económico liberal, con incentivos a la inversión extranjera, ha hecho que la inversión en el país aumente grandemente en los últimos años.

De acuerdo a Proinversion, al 31 de diciembre del 2015 había registrado un saldo de 24,233 millones de dólares de inversión extranjera directa; y de eso, un monto de 208.1 millones de dólares era la inversión china en el Perú ${ }^{7}$, que ubicaba a ese país como el $17^{\circ}$ inversor en importancia, aunque este monto no es toda la inversión de China en el Perú, como veremos después.

En todo caso, de acuerdo a Proinversion, del total de 208.1 millones de dólares de inversión china, 0.3 millones de dólares están en el sector comercio, 50 millones están en finanzas, y 157.8 millones de dólares están en minería 8 .

Las cifras de Proinversion no indican en su real magnitud el tamaño de la inversión extranjera en el país, pues solo registra la inversión de acuerdo al domicilio de las empresas, y varias empresas chinas, por ejemplo, invierten en el Perú domiciliadas en países como Reino Unido o Australia (la primera en el caso de la inversión china en Toromocho; la segunda en el caso de la inversión en Las Bambas, dos de las más grandes inversiones chinas en el Perú). Tampoco Proinversion registra la reinversión de las empresas extranjeras en el Perú (solo registra inversión nueva, de capital fresco, traído de fuera).

7 Ver Saldo de Inversión Extranjera Directa por País de Domicilio 1980 - Diciembre 2015 (Millones de US\$): http:/www.proinversion.gob.pe/modulos/LAN/landing.aspx?are=0\&pfl=1\&lan=10\&tit=proinver si\%C3\%B3n-institucional (accedido el 15 de febrero del 2016)

8 Ver Saldo de Inversión Extranjera Directa por País de Domicilio y Sector de Destino (Millones de US\$) $\mathrm{http} / /$ www.proinversion.gob.pe/modulos/LAN/landing.aspx?are $=0 \& \mathrm{pfl}=1 \&$ lan $=10 \&$ tit $=$ proinversi $\% \mathrm{C} 3$ \%B3n-institucional (accedido el 15 de febrero del 2016) 


\section{Pensamiento Crítico Vol. ᄅl $N^{\circ}$ I}

En Perú la inversión china en minería es la mayor entre todos los inversionistas en los últimos años. En el Gráfico 4 se ve que las empresas chinas tienen el 34\% o unos 19 mil millones de dólares de la cartera estimada de proyectos mineros en el Perú, estimada en 56 mil millones de dólares (a octubre del 2015).

\section{GRÁFICO4}

Cartera estimada de proyectos mineros según principal inversionista

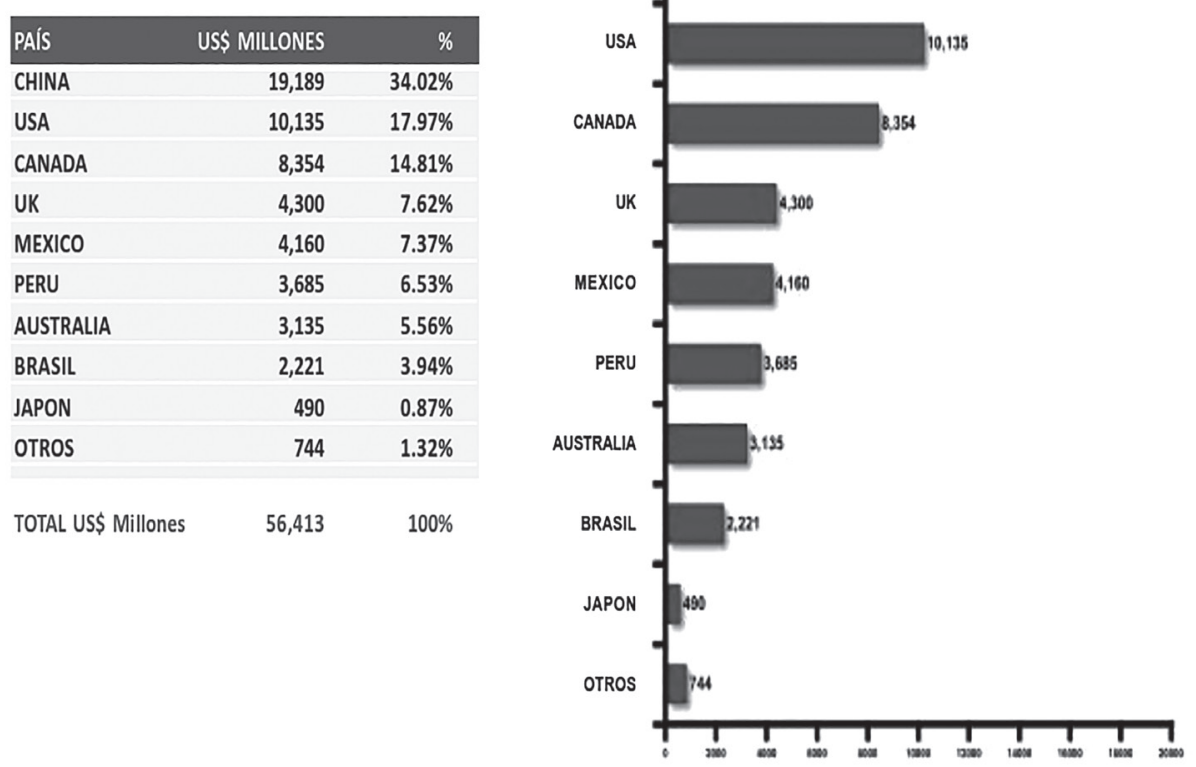

Fuente: Ministerio de Energía y Minas, ver Cartera Estimada de Proyectos Mineros: http://www.minem.gob.pe/_sector.php?idSector=1 (accedido el 12 de febrero del 2016)

Resumiendo, podemos ver que las empresas chinas que invierten en minería son las detalladas en el Cuadro 1. 


\section{Carlos Aquino Rodríguez}

CUADRO 1

Cartera estimada de principales proyectos mineros de empresas chinas

\begin{tabular}{|c|c|c|c|c|c|c|}
\hline EMPRESA & PROYECTO & REGIÓN & $\begin{array}{c}\text { MINERAL } \\
\text { PREDOMINANTE }\end{array}$ & $\begin{array}{c}\text { INICIO } \\
\text { ESTIMADO }\end{array}$ & $\begin{array}{c}\text { INVERSIÓN } \\
\text { EN MILLONES } \\
\text { DE US } \$\end{array}$ & $\begin{array}{c}\text { PRODUCCIÓN } \\
\text { POR ANNO } \\
\text { ADICIONAL }\end{array}$ \\
\hline $\begin{array}{c}\text { Shougang Hierro } \\
\text { Peru S.A.A. }\end{array}$ & $\begin{array}{c}\text { Ampliación } \\
\text { Marcona }\end{array}$ & Ica & $\mathrm{Fe}$ & Dic-18 & 1,500 & $3.5 \mathrm{Mill}$ TM \\
\hline $\begin{array}{c}\text { Minera Chinalco } \\
\text { Perú S.A. }\end{array}$ & $\begin{array}{c}\text { Ampliación } \\
\text { Toromocho }\end{array}$ & Junín & $\mathrm{Cu}$ & Ener-18 & 1,350 & $25,000 \mathrm{TMF}$ \\
\hline $\begin{array}{c}\text { Minera Las } \\
\text { Bambas S.A. * }\end{array}$ & Las Bambas & Apurímac & $\mathrm{Cu}$ & Mar-16 & 10,000 & $\begin{array}{c}450,00 \mathrm{TMF} / \mathrm{Cu} \\
\text { y 5,000 TMF/Mo }\end{array}$ \\
\hline $\begin{array}{c}\text { Minera Shouxin } \\
\text { PerúS.A.* }\end{array}$ & $\begin{array}{c}\text { Explotación } \\
\text { de relaves }\end{array}$ & Ica & $\mathrm{Cu}, \mathrm{Fe}, \mathrm{Zn}$ & 2016 & 239 & $\begin{array}{c}19,040 \mathrm{TMF} / \mathrm{Cu} \\
\text { y 29,988 TMF/Zn } \\
\text { y 80,580 TM/Fe }\end{array}$ \\
\hline $\begin{array}{c}\text { Jinzhao Mining } \\
\text { Peru S.A.* }\end{array}$ & $\begin{array}{c}\text { Pampa de } \\
\text { Pongo }\end{array}$ & Arequipa & $\mathrm{Fe}$ & Ener-18 & 1,500 & $15 \mathrm{Mill} \mathrm{TM}$ \\
\hline $\begin{array}{c}\text { JunfieldGroup } \\
\text { S.A.** }\end{array}$ & Don Javier & Arequipa & $\mathrm{Cu}$ & Por definir & 600 & Por definir \\
\hline $\begin{array}{c}\text { Lumina Copper } \\
\text { S.A.C.** }\end{array}$ & Galeno & Cajamarca & $\mathrm{Cu}, \mathrm{Mo}, \mathrm{Au}, \mathrm{Ag}$ & 2017 & 2,500 & $\begin{array}{c}350,000 \mathrm{TMF} / \mathrm{cu}, \\
82,000 \mathrm{Oz} / \mathrm{Au}, \\
2,500 \mathrm{TM} / \mathrm{Mo}, \\
2 \mathrm{Mill} \mathrm{Oz} / \mathrm{Ag}\end{array}$ \\
\hline $\begin{array}{c}\text { Rio Blanco } \\
\text { Copper S.A.** }\end{array}$ & Rio Blanco & Piura & $\mathrm{Cu}$ & 2019 & 1,000 & $200,000 \mathrm{TMF}$ \\
\hline
\end{tabular}

*Con E.I.A. Aprobado /En construcción **En exploración

Fuente: Ministerio de Energía y Minas, ver Cartera Estimada de Proyectos Mineros:

http://www.minem.gob.pe/_sector.php?idSector=1página 4 (accedido el 12 de febrero del 2016)

Se calcula que, con las grandes inversiones chinas en minería, sus empresas producirán un $33 \%$ del total de cobre en el Perú cuando las nuevas minas en Toromocho y Las Bambas produzcan a plena capacidad el 2017-2018 ${ }^{\circ}$. Otra empresa china produce el $100 \%$ del hierro en el país (la empresa Shougang es dueña de la única mina de ese mineral en el Perú, en Marcona).

Otro campo donde hay gran inversión china es en el energético. A través de la empresa Corporación Nacional de Petróleo China, CNPC, produciría un 30\% o más del petróleo y gas (tiene los lotes petrolíferos en Talara en el norte del Perú, y compró

9 Diario La República: "Un tercio del cobre peruano quedara en manos de China" http://larepublica.pe/15-

04-2014/un-tercio-del-cobre-peruano-quedara-en-manos-de-china (accesado 18 de febrero 2016) 


\section{Pensamiento Crítico Vol. ᄅl No I}

el 2014 el lote X en Piura, el lote 58, y casi la mitad del lote 57 en Cuzco de la empresa Petrobras $)^{10}$. CNPC pagó 2,600 millones de dólares por las operaciones de Petrobras en el Perú ${ }^{11}$. Según el diario El Comercio, la explotación del lote 58 demandará de CNPC un monto de inversión de 1,300 millones de dólares, y el gas que se obtenga de ahí es fundamental para la operación a largo plazo del Gasoducto Sur Peruano ${ }^{12}$.

Es interesante anotar que China fue el primer país que empezó a invertir en el Perú cuando este, en 1990,

se abre más al mundo y empieza un proceso de privatización de sus empresas públicas. Así, Shougang Corporation compra la mina Hierro Perú por 120 millones de dólares en 1992. Esa fue asimismo la primera incursión de una empresa china en el exterior, aparte de las inversiones que tenía en el Asia Oriental. China empieza así a expandirse por todo el mundo.

Una particularidad de la inversión china en el extranjero es que la gran mayoría es hecha por empresas estatales (últimamente hay inversión de algunas empresas privadas chinas, pero en la extracción de recursos naturales prácticamente todas son estatales).

Habría que señalar que varias empresas chinas mineras tienen problemas con su sindicato o la comunidad donde operan. Shougang todos los años enfrenta huelgas de su sindicato laboral en Marcona, Rio Blanco Copper hasta ahora no puede explorar pues la comunidad local se opone a esta porque teme que contamine el agua del lugar, $y$ Las Bambas últimamente está enfrentando problemas también con la comunidad local.

En pesquería, según un artículo publicado en el diario El Comercio, la empresa china Pacific Andes International Holdings ( $\mathrm{PAIH}$ ) controla la mayor cuota asignada de captura de pesca a través de las empresas CFG-Copeinca, con $16.8 \%$ del total. China Fishery Group (CFG) compró la empresa Copeinca el 2013 (por la cual pago 800 millones de dólares), pero actualmente tiene problemas y se habla de que podría vender sus activos

10 Ver artículo de Carlos Aquino y Luzmila Zanabria: "Las relaciones entre el Perú y China": https://www. academia.edu/19512218/Las_Relaciones_entre_el_Per\%C3\%BA_y_China

11 Ver Diario La República, 20 de mayo del 2014: "Ēl gigante asiático pisa fuerte en el Perú y Latinoamérica" http://larepublica.pe/20-05-2014/el-gigante-asiatico-pisa-fuerte-en-el-peru-y-america-latina

12 Ver Suplemento Día 1, página 10, Diario El Comercio, 18 de enero del 2016. 


\section{Carlos Aquino Rodríguez}

por unos 1,700 millones de dólares ${ }^{13}$. Uno de los posibles compradores podría ser el fondo de inversiones chino FOSUN, que en el 2013 intentó comprar a la Pesquera Diamante.

China es el principal comprador de harina de pescado del Perú y el año 2015 compró el $76.3 \%$ del total exportado ese año por el Perú ${ }^{14}$.

En el sector bancario, el banco chino Industrial and Commercial Bank of China, ICBC, el más grande del mundo por capitalización, opera desde el 2014, y ha realizado una inversión de 50 millones de dólares en el país. Otros bancos chinos, como el Bank of China, quieren ingresar al Perú pero no pueden pues la legislación peruana contempla que no puede haber dos bancos de un mismo dueño, algo que el gobierno chino pide cambiar ${ }^{15}$.

En el futuro está la inversión china en la probable implementación del ferrocarril bioceánico, que conectará el océano Atlántico con el Pacífico, uniendo al Perú y Brasil. Esta inversión podría demandar entre 10 mil millones de dólares y 30 mil millones de dólares. El estudio de factibilidad económica de este proyecto fue entregado por China en mayo de este año 2016, y se supone que también estaría listo el estudio de impacto ambiental a ser elaborado por los gobiernos de Perú y Brasil, por esa misma fecha.

\section{Perú como puente para China en Latinoamérica}

Perú es uno de los países en Latinoamérica que tiene una historia de relaciones antiguas con China. Fue el primero en recibir inmigrantes chinos a gran escala desde 1848 (se calcula que hasta 1874 llegaron unos 100 mil chinos al Perú) y el primero en Latinoamérica en establecer relaciones diplomáticas con el entonces Imperio chino, en 1874. En 1971 fue uno de los primeros en establecer relaciones diplomáticas con la nueva República Popular China.

13 Ver Suplemento Día 1, página 25, Diario El Comercio, 18 de enero del 2016.

14 Ver Reporte de Comercio Bilateral Perú-China, diciembre 2015, MINCETUR, http://www.mincetur.gob. pe/newweb/Default.aspx?tabid=793 (accedido el 15 de febrero del 2016).

15 Ver Agencia Peruana de Noticias Andina, edición online: "Two Chinese Banks keen to enterfinancialmarket in Peru" http://www.andina.com.pe/ingles/noticia-two-chinese-banks-keen-to-enter-financial-marketin-peru-574595.aspx (accedido, 15 de febrero del 2016). En China los bancos son del gobierno chino, así que todos tienen un mismo dueño. 


\section{Pensamiento Crítico Vol. ᄅl No I}

Hay una gran comunidad de origen chino en el Perú, la más grande de Latinoamérica. Se calcula que quizás $5 \%$ de la población peruana tiene origen chino (algunos dicen que hasta un $10 \%)$.

En todo caso, Perú es uno de los países con un régimen económico bastante abierto a la inversión extranjera y el comercio. Es uno de los 3 países que tiene tratado de libre comercio con China en Latinoamérica (los otros son Chile y Costa Rica). Como se vio en el acápite I, en Perú la inversión china en minería es la mayor en los últimos años; y en otros sectores, como en hidrocarburos y pesquería, cada vez tiene una mayor presencia.

Desde hace varios años China es el mayor socio comercial del Perú y desde el año pasado es la mayor fuente de sus importaciones también, desplazando a EE.UU. El Perú exportó en el 2015 por un monto de 7,333 millones de dólares e importó de China unos 8,661 millones de dólares. Las exportaciones a China aumentaron de 7,043 millones de dólares en el 2014, a pesar de que las exportaciones totales del Perú al mundo cayeron de 38,641 millones de dólares en el 2014 a 32,646 millones de dólares en el 2015; y las importaciones desde China disminuyeron un poco desde 8,918 millones de dólares el 2014, pero las importaciones totales del Perú cayeron mucho más, desde 42,184 millones de dólares el 2014 hasta 38,066 millones de dólares el 2015. ${ }^{16}$

Dada la posición geográfica del Perú en Sudamérica, en el medio de esta y con cara al océano Pacífico, puede convertirse en nexo entre China y los países de la región, en un hub para el capital chino en toda Latinoamérica, pero para esto el Perú necesita mejorar su infraestructura física (con mejores puertos, aeropuertos, carreteras, etc.), conectividad y, según los chinos, cambiar algunas cosas en la legislación. Como se vio, por ejemplo, China quiere tener otro banco más en el Perú, aparte del ICBC, pero no puede ahora.

16 Ver Reporte de Comercio Bilateral Perú-China, diciembre 2015, MINCETUR, http://www.mincetur.gob. pe/newweb/Default.aspx?tabid $=793$ 


\section{Carlos Aquino Rodríguez}

\section{Planes de China para invertir en otros proyectos aparte de la extracción de recursos naturales: infraestructura física como ferrocarriles, energía atómica, agricultura, etc.}

China está interesada en financiar el proyecto del tren bioceánico pues este tren permitiría sacar recursos mineros, agropecuarios, forestales desde Brasil al Pacífico, de una manera más rápida y económica, que si tuvieran que ir desde Brasil por el Canal de Panamá. Asimismo, China está interesada en vender su tecnología de trenes, y eso lo puede financiar. De hecho, China está compitiendo en varios países del mundo vendiendo su tecnología de trenes con financiamiento barato, más atractivo que el que ofrece la competencia, como se vio cuando el año pasado, en setiembre, ganó la licitación a Japón, que estaba confiado en ganar, para hacer el tren rápido de Yakarta a Bandung, en Indonesia ${ }^{17}$.

China ya ha proveído de vagones para algunas líneas de metro en Río de Janeiro ${ }^{18}$. China también ha ofrecido construir la segunda fase del metro de Quito por 1,600 millones de dólares, financiado a 30 años y a una tasa de interés de $2 \%^{19}$. Empresas chinas han postulado para construir la línea de metro en Panamá, que no ganaron pero insisten, y ganaron una licitación en México para construir un tren bala, que luego se suspendió ${ }^{20}$, lo que hubiera sido el primer tren bala en Latinoamérica.

Algunos son escépticos de que el proyecto del tren bioceánico chino se complete, como al parecer ocurre con el proyecto de un empresario chino para construir un canal en Nicaragua para competir con el canal de Panamá. En este caso, el proyecto es privado, y la diferencia es que el tren bioceánico es un proyecto del gobierno chino. Se cita a veces como razones para que algunos proyectos anunciados por China no se completen las diferentes velocidades entre el accionar del gobierno chino y de los gobiernos latinoamericanos, donde los segundos son más lentos por trabas burocráticas. También se

17 Ver Nikkei Asian Review: "Japan underestimated China over Indonesia train project", November 10, 2015 http://asia.nikkei.com/Politics-Economy/International-Relations/Japan-underestimated-China-overIndonesian-train-project(accedido, 14 de febrero del 2016)

18 Ver CCTV. com en español "Metro hecho en China prestara servicios en JJ.OO. de Rio 2016"http://espanol.cntv.cn/2015/05/22/VIDE1432265167181566.shtml(accedido, 14 de febrero del 2016)

19 Ver Ecuavisa, marzo 2015: "China propone construir el Metro de Quito" http://www.ecuavisa.com/articulo/noticias/actualidad/101234-china-propone-construir-metro-quito(accedido el 15 de febrero del 2016)

20 Ver La República: "Firma China construirá en México el primer tren bala de América Latina"http:/www.larepublica.co/firma-china-construir\%C3\%A1-en-m\%C3\%A9xico-el-primer-tren-bala-de-am\%C3\%A9ricalatina_188261(accedido, 14 de febrero del 2016) 


\section{Pensamiento Crítico Vol. ᄅl $\mathrm{N}^{\circ} \mathrm{I}$}

menciona la falta de proyección a largo plazo de los gobiernos en Latinoamérica y el problema de la corrupción ${ }^{21}$.

En el campo de la energía nuclear, Argentina, que fue el primer país en instalar en Latinoamérica una planta de energía nuclear en $1974^{22}$, Atucha 1, ha firmado con China contratos para hacer plantas nucleares. En el siguiente cuadro se ven los reactores que Argentina tiene en operación en la actualidad.

\section{CUADRO 2}

Reactores de energía nuclear en Argentina en operación ${ }^{23}$

\section{Operating Argentine nuclear power reactors}

\begin{tabular}{|lllll|}
\hline Reactor & Location & Model & Net MWe & First power \\
\hline Atucha 1/Peron & $\mathbf{1 0 0 ~ k m ~ N W}$ of Buenos Aires & PHWR (Siemens) & 335 & 1974 \\
\hline Atucha 2/Kirchner & & PHWR (Siemens) & 692 & June 2014 \\
\hline Embalse & Córdoba & PHWR (CANDU-6) & 600 & 1983 \\
\hline Total (3) & & & $1627 \mathrm{MWe}$ & \\
\hline
\end{tabular}

Net MWe from PRIS

Fuente: http://world-nuclear.org/information-library/country-profiles/countries-a-f/argentina.aspx (accedido, 14 de febrero del 2016)

Como se ve en el cuadro siguiente el cuarto reactor, el Atucha 3, se construirá por un contrato firmado en noviembre del 2015 con China, que lo hará a través de la empresa China National Nuclear Corporation, CNNC. Además, hará un quinto reactor, por un valor total de 15 mil millones de dólares, para el cual el país asiático proveerá un $85 \%$ del financiamiento ${ }^{24}$. El préstamo se pagaría en 18 años y la tasa de interés estaría por debajo del $6.5 \%$.

El Atucha 3 es un modelo "pressurized heavy-water reactor" (PHWR). El quinto reactor, que sería proveído por China, será del Modelo Hualong One, que suministraría

21 CNN Money: "China's big bet on Latin America is going bust" http://money.cnn.com/2016/02/16/news/ economy/china-latin-america-projects-fail/ (accedido, 17 de febrero del 2016)

22 Power-Techonoly.com: "Atucha Nuclear PowerPlant, Argentina": http://www.power-technology.com/projects/atucha/ (accedido, 14 de febrero del 2016)

23 World Nuclear Association: "Nuclear Power in Argentina": http://world-nuclear.org/information-library/ country-profiles/countries-a-f/argentina.aspx (accedido, 14 de febrero del 2016)

24 Ver nota 23. 


\section{Carlos Aquino Rodríguez}

la mayor cantidad de energía hasta el momento: unos 1950 MWe. Cuando se terminen, estas dos plantas proveerían el doble de la capacidad de energía nuclear que suministran las tres plantas existentes. El reactor en Atucha 3 usará la tecnología nuclear canadiense "Candu" y costaría unos 6 mil millones de dólares. El modelo Hualong One es de tecnología china y es el que este país está promoviendo para el mercado externo ${ }^{25}$.

\section{CUADRO 3}

Reactores de energía nuclear en Argentina bajo construcción, planeado y propuestos

\begin{tabular}{|c|c|c|c|c|c|}
\hline Reactor & Location & Model & Gross MWe & $\begin{array}{l}\text { Construction } \\
\text { start }\end{array}$ & First power \\
\hline Carem-25 & $100 \mathrm{~km} \mathrm{NW}$ of Buenos Aires & CAREM & 27 & Feb 2014 & 2018 \\
\hline \multicolumn{6}{|c|}{ Under construction: 1} \\
\hline Atucha 3 & $100 \mathrm{~km} \mathrm{NW}$ of Buenos Aires & PHWR (EC6?) & $800 ?$ & $2016 ?$ & ? \\
\hline 5th unit & $\begin{array}{l}100 \text { km NW of Buenos Aires?, } \\
\text { Paraguay River? }\end{array}$ & Hualong One & 1150 & $2017 ?$ & $?$ \\
\hline \multicolumn{3}{|l|}{ Planned: 2} & \multicolumn{3}{|l|}{$1950 \mathrm{MWe}$} \\
\hline$?$ & Formosa, on Paraguay River & CAREM & $100-200 ?$ & & $?$ \\
\hline$?$ & $?$ & VVER-1200 & 1200 & & $2023 ?$ \\
\hline \multicolumn{3}{|c|}{ Proposed: 2} & \multicolumn{3}{|l|}{$1300 \mathrm{MWe}$} \\
\hline
\end{tabular}

Fuente: http://world-nuclear.org/information-library/country-profiles/countries-a-f/argentina.aspx (accedido, 14 de febrero del 2016)

China está impulsando la venta de su tecnología nuclear al mundo. Durante la visita del presidente Xi Jinping a Inglaterra en octubre pasado, este firmó un acuerdo para vender a ese país sus reactores, en un proyecto liderado por Francia. Se dice que China ha identificado unos 60 países como potenciales clientes y espera vender unas 30 plantas nucleares, de las 200 que se espera se construirán en esos países de aquí al $2030^{26}$.

En el sector agrícola se habla bastante de la intención por parte de China de comprar o alquilar tierra agrícola en la región. De hecho, China necesita alimentos de la región y no solo quiere comprar, sino cultivarlos allí y llevárselos luego. Pero el tema es sensitivo

25 Ver Financial Times web site: "China to build two nuclear plants in Argentina in a \$15 bndeal" http:// www.ft.com/intl/cms/s/0/2d264e78-8cf9-11e5-a549-b89a1dfede9b.html\#axzz40MEhctoU (accedido, 16 de febrero del 2016)

26 Ver Nota 25 


\section{Pensamiento Crítico Vol. 己l Nº}

y ha habido protestas en algunos lugares cuando intentó comprar largas extensiones de terreno.

Según un estudio ${ }^{27}$, China ya ha comprado tierra en algunos países de Latinoamérica. Por ejemplo, el 2011 compró en Jamaica unas 27,800 hectáreas para sembrar caña de azúcar, y el 2010 en Bolivia, Santa Cruz, en el Proyecto de Zona Industrial para Soya, una empresa china, Pengxin Group Co., acordó explotar 12,488 hectáreas.

Hay algunas operaciones de compra o alquiler de tierras por parte de China no confirmadas o estancadas. Una de las más controversiales fue la intención de comprar primero y alquilar después unas 300,000 hectáreas en Argentina, en la región de Río Negro, para sembrar soya, maíz y trigo. El 2011 la empresa china Beidahuang firmó un acuerdo para ese objetivo con el gobierno provincial de Río Negro, que podría implicar una inversión de 1,500 millones de dólares, pero hasta ahora, al parecer, no se concreta la operación ${ }^{28,29}$.

La compra o alquiler de tierras por extranjeros es muy sensitiva en muchos países, por eso algunas operaciones no se han llegado a concretar.

En todo caso, hay que recordar que China el año pasado, durante la visita a Latinoamérica de su primer ministro, Li Keqiang, estableció 3 fondos para el financiamiento de la industria y la infraestructura de la región. "El mayor de los fondos, el Fondo China-LAC de Inversión para la Cooperación Industrial cuenta con 20,000 millones de USD" 30 .

27 The Inter-American Dialogue, China and Latin America Report, June 2015, Margaret Myers and GuoJie: "China's Agricultural Investment in Latin America: A critical assessment", pagina 12

28 Ver Agencia de Datos y Noticias: "China quiere soja, ríos, tierra y un puerto propio en la Patagonia" http:// adnagencia.info/el-pais/item/4676-china-viene-por-la-soja,-r\%C3\%ADos,-tierra-y-un-puerto-propio-enla-patagonia.html (accedido, 16 de febrero del 2016)

29 VerThe Inter-American Dialogue, China and Latin America Report, June 2015, Margaret Myers and GuoJie: "China's Agricultural Investment in Latin America: A critical assessment", pagina 10

30 Ver Diálogo Chino: "China aumenta préstamos a Latinoamérica" http://dialogochino.net/china-aumentaprestamos-a-america-latina/?lang=es(accedido el 16 de febrero del 2016) 


\section{Carlos Aquino Rodríguez}

\section{Conclusiones}

China seguirá necesitando de recursos naturales y alimentos para su población de 1,360 millones de personas. La región latinoamericana ofrece esos recursos, además de un mercado de 600 millones de habitantes y en crecimiento.

China tiene un plan para poder incrementar su relación económica con la región, de mayor comercio, inversión y cooperación económica. Es también el objetivo de las empresas chinas internacionalizarse, competir en el extranjero invirtiendo ahí, y así poder disponer de la capacidad instalada en exceso que tiene en su país algunas de sus industrias debido a la desaceleración de su economía (como las de acero, aluminio, automóviles, petroquímica, ciertos tipos de maquinarias, plástico, etc. $)^{31}$.

Si bien Perú tiene una estrategia en su relación con China, la mayoría de los países latinoamericanos parece que no. La mayor parte de las iniciativas para una mayor relación económica vienen de China. Hay un desconocimiento acerca de ese país por parte de los latinoamericanos, mientras que los chinos, que si bien es cierto no conocen plenamente la región, se preparan. Los gobiernos de la región además tienen una visión de corto plazo, y los chinos planifican para el mediano y largo plazo.

Una desaceleración mayor de la economía china podría afectar la capacidad del gobierno chino de financiar proyectos en la región, pero esto solo implicará un proceso más lento, porque definitivamente el comercio e inversión de China con Latinoamérica seguirá aumentando.

\section{Referencias bibliográficas}

CEPAL. "China Bets On Strategic Ties with Latin America and the Caribbean". Disponible en: http://www.cepal.org/en/pressreleases/china-apuesta-por-vinculoestrategico-con-america-latina-y-el-caribe

CEPAL (2015). "Latin America and the Caribbean and China: towards a new era in economic cooperation”. Disponible en: http://www.cepal.org/en/publications/38197latin-america-and-caribbean-and-china-towards-new-era-economic-cooperation

31 http://www.chinadaily.com.cn/business/2016-02/20/content_23564138.htm 


\section{Pensamiento Crítico Vol. 己l NoI}

Ministerio de Energía y Minas: Cartera Estimada de Proyectos Mineros. Disponible en: http://www.minem.gob.pe/_sector.php?idSector $=1$

MINCETUR (2015). Reporte de Comercio Bilateral Perú-China.Disponible en: http:// www.mincetur.gob.pe/newweb/Default.aspx?tabid $=793$

Margaret Myers and Guo Jie (2015). "China's Agricultural Investment in Latin America: A critical assessment". The Inter-American Dialogue, China and Latin America Report. 